\title{
Notas sobre la dieta y el hábitat del Gorrión-Montés paisa Atlapetes blancae (Passerellidae)
}

\author{
Notes on the Diet and Habitat of Antioquia Brush-finch Atlapetes blancae (Passerellidae)
}

Sergio Chaparro-Herrera ${ }^{1,2,3 *}$, Mateo Hernández-Schmidt ${ }^{1,4}$, Andrea Lopera-Salazar ${ }^{1,2}$

\begin{abstract}
Resumen
Parte de los datos más relevantes y básicos para implementar medidas de conservación enfocadas en una especie, requieren de no más que un metódico registro de las observaciones. Presentamos los datos de dieta y estrategias de forrajeo de Atlapetes blancae, recopilados entre diciembre de 2018 y enero de 2020 en San Pedro de los Milagros, Santa Rosa de Osos y Llanos de Cuivá en Yarumal, Antioquia, Colombia. Presentamos también una descripción de su hábitat y comentarios sobre las estrategias posibles para implementar acciones de conservación. Esta información no solo aporta al conocimiento biológico de la especie, sino que también ofrece información crucial sobre la vegetación relacionada con $A$. blancae para que sea incluida en acciones de restauración ecológica, evitando así la pérdida del hábitat de esta ave endémica que se encuentra en la categoría máxima de amenaza a la extinción.
\end{abstract}

Palabras clave: Antioquia, Colombia, conservación, endémica, historia natural, peligro crítico

\begin{abstract}
Some of the most relevant and basic data for implementing species-focused conservation measures require nothing more than a methodical record of observations. We present data on the diet and feeding strategies of Atlapetes blancae collected between December 2018 and January 2020 in San Pedro de los Milagros, Santa Rosa de Osos, and Llanos de Cuivá in Yarumal, Antioquia, Colombia. We also present a description of their habitat and comments on possible strategies to implement conservation actions. This information not only contributes to the biological knowledge of the species, but also offers crucial information about the vegetation related to $A$. blancae so that it can be included in the ecological restoration actions and thus avoid the loss of the habitat of this endemic bird that is in the highest category of threat of extinction.
\end{abstract}

Keywords: Antioquia, Colombia, conservation, critically endangered, endemic, natural history

\footnotetext{
1. Proyecto Atlapetes: Ecología y Conservación del Gorrión-Montés Paisa (Atlapetes blancae), Antioquia, Colombia.

2. Grupo de Ecología y Evolución de Vertebrados, Universidad de Antioquia, Medellín, Colombia.

3. Laboratorio de Ecología Evolutiva y Urbana, Universidad del Norte, Barranquilla, Colombia.

4. Consultor independiente.

* Autor para correspondencia: <sergioupn@gmail.com>
} 
Conocer la información básica sobre la historia natural y la ecología de una especie, es un paso imprescindible cuando se quiere avanzar hacia su conservación (Lees et al., 2020). A su vez, la necesidad de emprender acciones para conservar especies es resultado de una crisis medioambiental de la que nos hemos percatado en las últimas décadas y que nos ha llevado a clasificar las especies en niveles de riesgo a la extinción. Perder especies puede ser el resultado de haber perdido hábitats, la historia que las relaciona a sus áreas de distribución y todos los servicios ecosistémicos y el bienestar que los sistemas naturales proveen para la vida en el planeta. Todas las especies tienen una estrecha relación con el espacio geográfico y además responden a factores históricos y ambientales, por lo que surge especialmente la necesidad de proteger aquellas que presentan alguna categoría de endemismo o rangos de distribución restringidos (Chaparro-Herrera et al., 2013). Este es el caso del Gorrión-Montés paisa (Atlapetes blancae Donegan, 2007), una especie endémica de Colombia y catalogada como en Peligro Crítico (CR) (BirdLife International, 2018; Chaparro-Herrera et al., 2013; Renjifo et al., 2014), que presenta a la fecha grandes vacíos de información puesto que solo se conocía de tres especímenes colectados, únicamente uno de ellos con fecha, 1971 y que fueron a su vez la base para su descripción formal en 2007 (Donegan, 2007). Sin embargo, luego de su descripción, A. blancae fue buscada en el municipio de San Pedro de los Milagros, departamento de Antioquia, zona de donde presuntamente provenían los especímenes, sin lograr encontrarla (Correa et al., 2019; Donegan et al., 2009). Solo hasta el 2018, cuando se redescubrió en este mismo municipio (Correa et al., 2019), fue posible empezar a conocer aspectos básicos de su biología como hábitat, distribución, vocalizaciones, reproducción, entre otros (Chaparro-Herrera y LoperaSalazar, 2019; Correa et al., 2019). A continuación, presentamos los registros de alimentación y estrategias de forrajeo de esta especie, recopilados desde diciembre de 2018 hasta enero de 2020, en varias localidades de su distribución al norte de la cordillera Central en el área denominada Altiplano de Santa Rosa de Osos, en el departamento de Antioquia, mediante observaciones libres enmarcadas en el Proyecto Atlapetes (https://arcg.is/1yz0eK), así como algunas notas de su hábitat y aspectos relevantes de restauración ecológica.

\section{Área de estudio}

El Altiplano de Santa Rosa de Osos-Rionegro, perteneciente a la región fisiográfica denominada "Altiplanos de Santa Rosa de Osos, Rionegro y Sonsón", se encuentra ubicado al extremo norte de la cordillera Central, en el departamento de Antioquia, en el batolito antioqueño (cuerpo ígneo de gran tamaño originado en el Cretáceo superior) (Arias, 1995; Arias, 2011; Arias et al., 2000; Arias y Gonzáles, 2007). El altiplano se encuentra dividido en dos por la formación del Valle de Aburrá y el cañón del río Medellín: Altiplano de Santa Rosa de Osos en el norte, localizado entre los 2300 y los 3000 m s. n. m. y Altiplano de Rionegro, al sur, ubicado entre los 2200 y los 2300 m s. n. m. (Arias, 2011; Instituto Geográfico Agustín Codazzi, 2007) (figura 1); por lo anterior, en este artículo solo nos referiremos al Altiplano de Santa Rosa de Osos (ASRO), debido a que $A$. blancae, a la fecha, solo ha sido registrado en la porción norte de este altiplano (Chaparro-Herrera y Lopera-Salazar, 2019; Correa et al., 2019).

El ASRO se encuentra dominado por un clima frío muy húmedo y está compuesto por colinas intermedias y bajas y un sistema de bloques colinados escalonados (Arias et al., 2006; Arias, 2011; Arias y Gonzáles, 2007). Presenta tres tipos de relieve que conforman la estructura mórfica de este segmento: altiplanos, escarpes regionales y cañones, elementos que se han originado en respuesta a un proceso discontinuo de levantamiento tectónico de la cordillera Central y el cual se remonta hasta antes del Oligoceno medio (Arias et al., 2000; Arias, 2011). En cuanto a sus suelos, estudios realizados indican la presencia de oxisoles, spodosoles (subgrupo troporthod), formados en depósitos de escorrentía cuarcíticos, que se encuentran por encima de la discordancia erosiva superior, presentes en cimas planas y en rampas de las colinas, en especial, en la parte norte del altiplano en el sector de Llanos de Cuivá (Arias et al., 2000; Arias, 2011).

\section{Dieta}

San Pedro de los Milagros: entre diciembre de 2018 y enero de 2020, en ocho localidades entre los 2493 
y $\operatorname{los} 2837 \mathrm{~m}$ s. n. m., (figura 2A) registramos en matorrales el consumo de: Berberis medellinensis (Berberidaceae), Weinmannia pubescens (Cunoniaceae), Vaccinium meridionale, Bejaria aestuans (Ericaceae) y Baccharis nitida (Asteraceae) (figuras 3 y 4, anexo 1). Sumado a esto se observó a $A$. blancae consumiendo insectos y larvas en algunas de las plantas mencionadas anteriormente y en un arbusto de Solanum sp. (Solanaceae). Estos registros se suman a los ya publicados por Correa et al. (2019) en este municipio, entre enero y noviembre de 2018: consumo de frutos y semillas de Persicaria nepalensis (Polygonaceae), W. pubescens (Cunoniaceae), Miconia $\mathrm{cf}$. theizans (Melastomataceae), V. meridionale (Ericaceae), Viburnum undulatum (Viburnaceae) y Myrcianthes sp. (Myrtaceae); y entre abril y mayo de 2019 por Chaparro-Herrera y Lopera-Salazar (2019) durante etapa de anidación: B. nitida, B. aestuans, $W$. pubescens, néctar de Elleanthus aurantiacus (Orchidaceae) (figura 3, anexo 1), y el consumo de larvas e insectos adultos.

Santa Rosa de Osos: entre septiembre y noviembre de 2019, en dos localidades entre los 2660 y los 2765 m s. n. m., registramos en matorrales (figura 2B) el consumo de: Drimys granadensis (Winteraceae),
Monnina sp. (Polygalaceae), Cavendishia bracteata (Ericaceae), B. aestuans, B. nitida, y el consumo de insectos y larvas en algunas de las plantas mencionadas anteriormente (figura 3, anexo 1).

Yarumal (sector Llanos de Cuivá): el 10 de mayo de 2019 observamos en un área de páramo azonal y matorrales asociados, a $2740 \mathrm{~m} \mathrm{~s} . \mathrm{n}$. m. (figura 2C, 2D, anexo 1), el consumo de Hesperomeles obtusifolia (Rosaceae), Psychotria sp. (Rubiaceae), B. nitida, Disterigma alaternoides y Gaultheria erecta (Ericaceae), además de registrar el consumo de insectos y larvas. En este caso se trató de la observación de una pareja de adultos y un individuo juvenil, el cual en dos ocasiones fue alimentado por sus padres con frutos de H. obtusifolia.

En el caso del consumo de plantas, las estrategias de forrajeo fueron variadas, entre las que encontramos (categorías y definiciones tomadas de Villareal et al., 2006): picoteo, agacharse, estirarse, empinarse, brincar a los lados, brincar arriba y salida a recoger; por su lado, el consumo de insectos y larvas se dio mediante técnicas como remoción de hojas en el suelo, picoteo, introducir pico, abrir sustrato, estirarse, empinarse y brincar a los lados. A con-
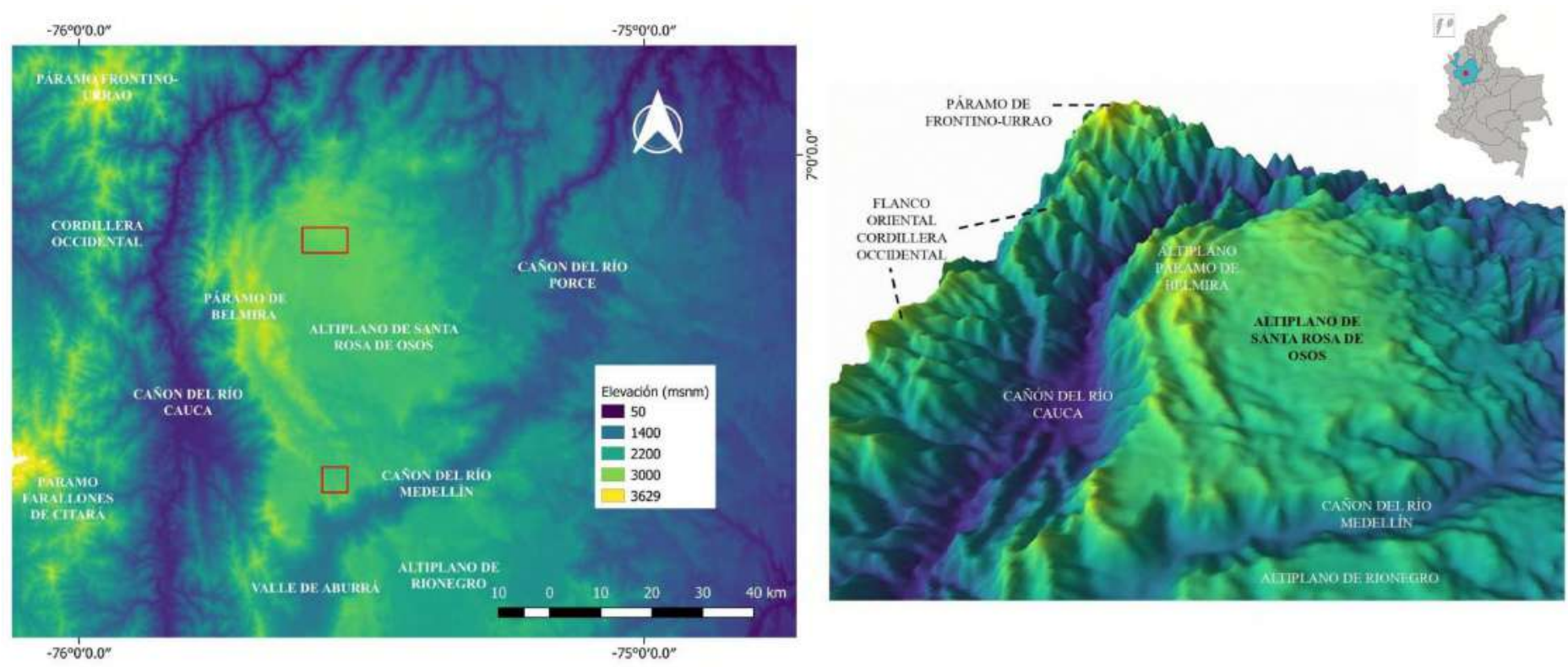

Figura 1. Ubicación espacial y morfología del Altiplano de Santa Rosa de Osos, porción norte de la cordillera Central de Colombia. 
tinuación, resaltamos algunos comportamientos de forrajeo registrados:

- En el consumo de néctar de E. aurantiacus, las flores de esta eran arrancadas y mantenidas en el pico girándolas en repetidas ocasiones, posiblemente tratando de succionar o extraer el néctar para luego dejar caer las flores completas al suelo. Este comportamiento fue observado en repetidas ocasiones en San Pedro de los Milagros, entre abril y mayo de 2019, durante la época de incubación.

- Cuando el individuo joven fue alimentado en dos ocasiones por sus padres con frutos $H$. obtusifolia, en mayo de 2019 en el municipio de Yarumal, este individuo giró los frutos en su pico extrayendo la pulpa y dejando caer el epicarpio al suelo.

- En el consumo de insectos destacamos el comportamiento registrado en el municipio de Yarumal en mayo de 2019, que consistió en el picoteo constante de una rama gruesa hueca, rasgando la corteza en repetidas ocasiones, para extraer insectos que se encontraban en su interior (ver: https://flic.kr/p/ 2jXBti2).

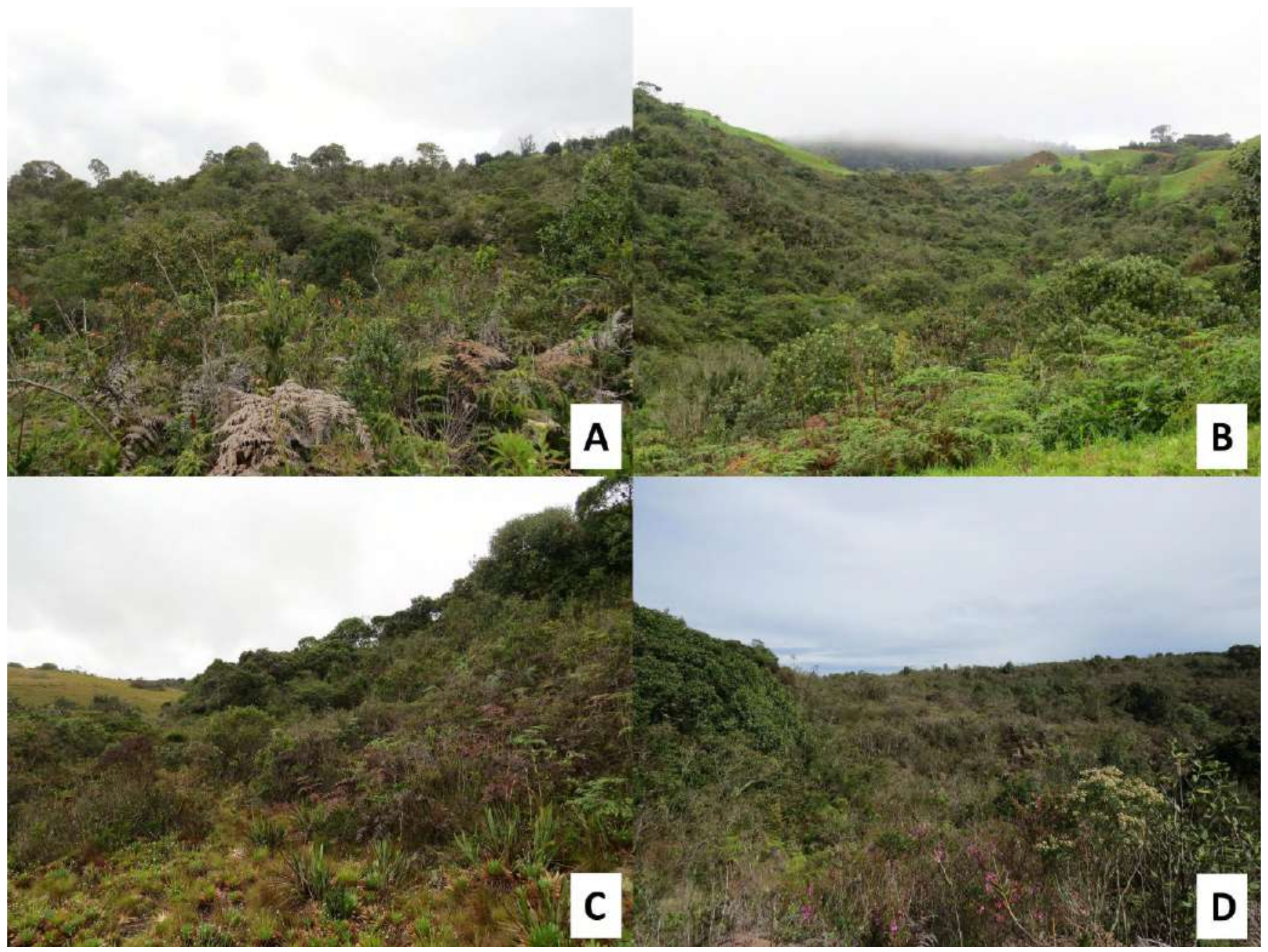

Figura 2. Hábitat de Atlapetes blancae en el altiplano de Santa Rosa de Osos, departamento de Antioquia, Colombia. A. Matorrales del municipio de San Pedro de los Milagros, B. Matorrales del municipio de Santa Rosa de Osos. C y D. Páramo azonal y matorrales asociados en el municipio de Yarumal, sector Llanos de Cuivá. Fotografías: Sergio Chaparro-Herrera. 


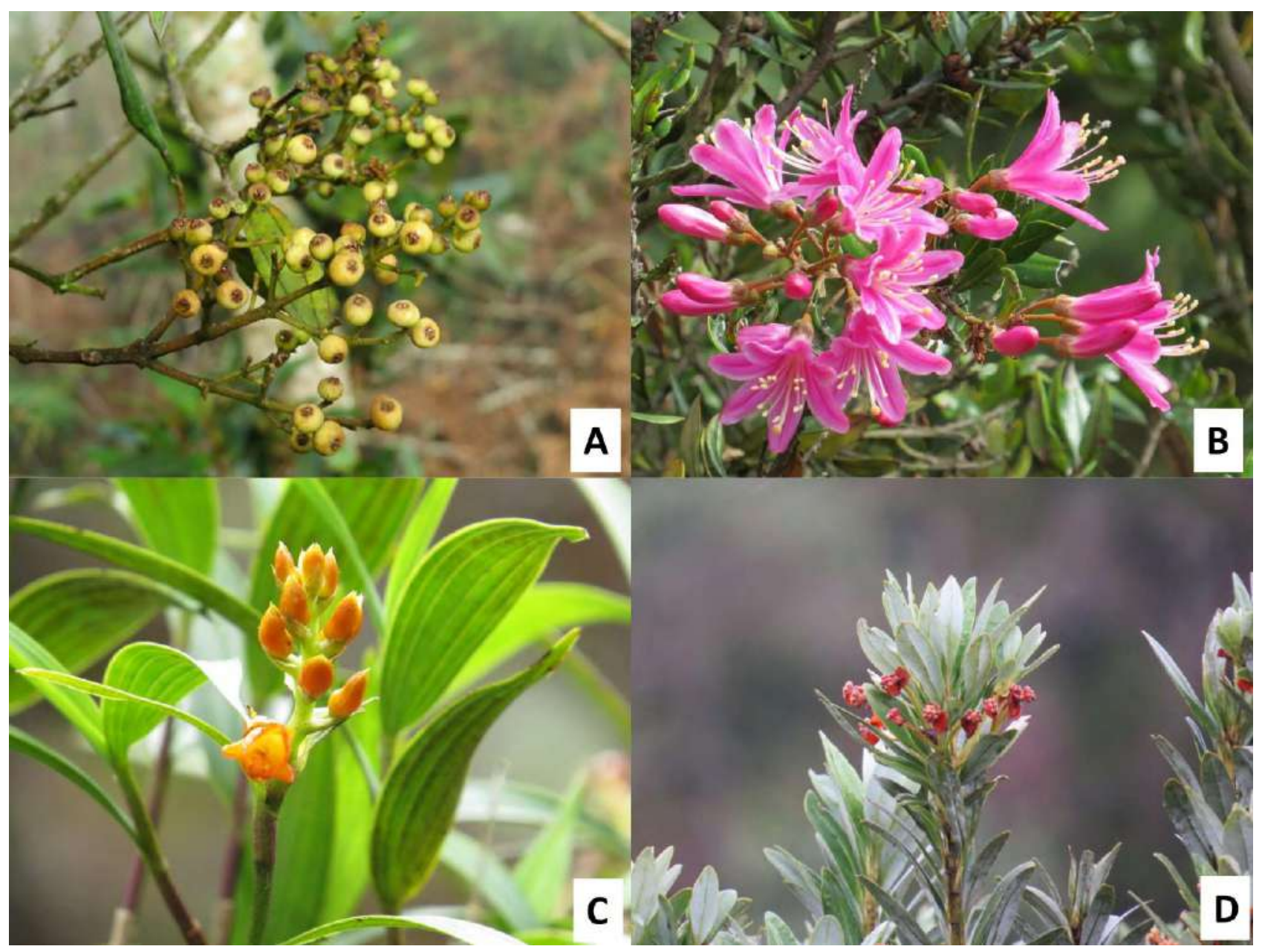

Figura 3. Algunas de las especies consumidas por Atlapetes blancae en el altiplano de Santa Rosa de Osos, departamento de Antioquia, Colombia. A) Miconia cf. theizans, B) Bejaria aestuans, C) Elleanthus aurantiacus, D) Drimys granadensis. Fotografías: Sergio Chaparro-Herrera.

\section{Hábitat}

Vegetación: la vegetación que en el pasado cubrió la mayor parte de las montañas del ASRO, así como la de otras regiones altoandinas del departamento de Antioquia, eran bosques montanos maduros, donde el roble (Quercus humboldtii) usualmente era la especie arbórea dominante (Espinal, 2011; Toro, 2010). En la actualidad, el municipio de San Pedro de los Milagros se encuentra dominado por un paisaje de potreros para la ganadería, que conforma el uso actual de más del $70 \%$ del territorio (Administración Municipal, 2000). En estos paisajes, los árboles más altos suelen ser especies exóticas (acacias, eucaliptos, pinos, cipreses), los cuales crecen dispersos, en pe- queñas plantaciones o formado franjas a lo largo de linderos y caminos. También se encuentran pequeños relictos de vegetación nativa, sobre todo matorrales y bosques secundarios.

La vegetación en la que habita $A$. blancae, en el municipio de San Pedro de los Milagros y Santa Rosa de Osos, consiste en matorrales y bosques de bajo porte (figura 2A, 2B) (zona de vida de Bosque muy húmedo Montano Bajo (bmh-MB) [Espinal, 2011; Holdridge, 1967]), los cuales pueden desarrollarse en forma natural en lugares con limitantes para el desarrollo de bosques de mayor porte; por ejemplo, sobre suelos pobres en nutrientes, ácidos, superficiales, pedregosos o en afloramientos rocosos (Avellaneda- 
Cadena y Betancur, 2007). Estos matorrales también pueden nacer al ser degradados la vegetación y los suelos en ambientes antes cubiertos con bosques más extensos, por ejemplo, en sitios donde ha habido extracción continúa de maderas, deforestación, minería y construcción de carreteras (Fernández-Méndez et al., 2016; Salamanca y Camargo, 2000).

En San Pedro de los Milagros estos matorrales están dominados por una vegetación arbustiva, de hasta 3-6 m de altura, compuesta por especies como Ageratina glyptophlebia, B. nitida (Asteraceae), M. theizans, Tibouchina grossa, T. mollis (Melastomataceae), Myrica pubescens, M. parvifolia (Myricaceae), Pteridium arachnoideum (Dennstaedtiaceae), B. aestuans, C. bracteata, V. meridionale, (Ericaceae), H. obtusifolia (Rosaceae), Lepechinia bullata
(Lamiaceae) y Viburnum anabaptista (Viburnaceae), entre otras. A su vez, dispersos entre el matorral o formando grupos en algunos sectores, se encuentran algunos árboles de mayor porte como Clusia multiflora (Clusiaceae) y W. pubescens (Cunoniaceae), que a veces pueden formar pequeños parches de bosque de hasta $12 \mathrm{~m}$ de altura, junto con Q. humboldtii (Fagaceae), que antiguamente fue el árbol dominante y que ya es escaso en el área.

La vegetación mencionada de matorrales y bosque denso de porte bajo se desarrolla en condiciones típicas de la alta montaña, donde los suelos suelen ser superficiales, pedregosos, expuestos a la radiación solar, al viento y a fuertes cambios de temperatura (Ferreyra et al., 1998).

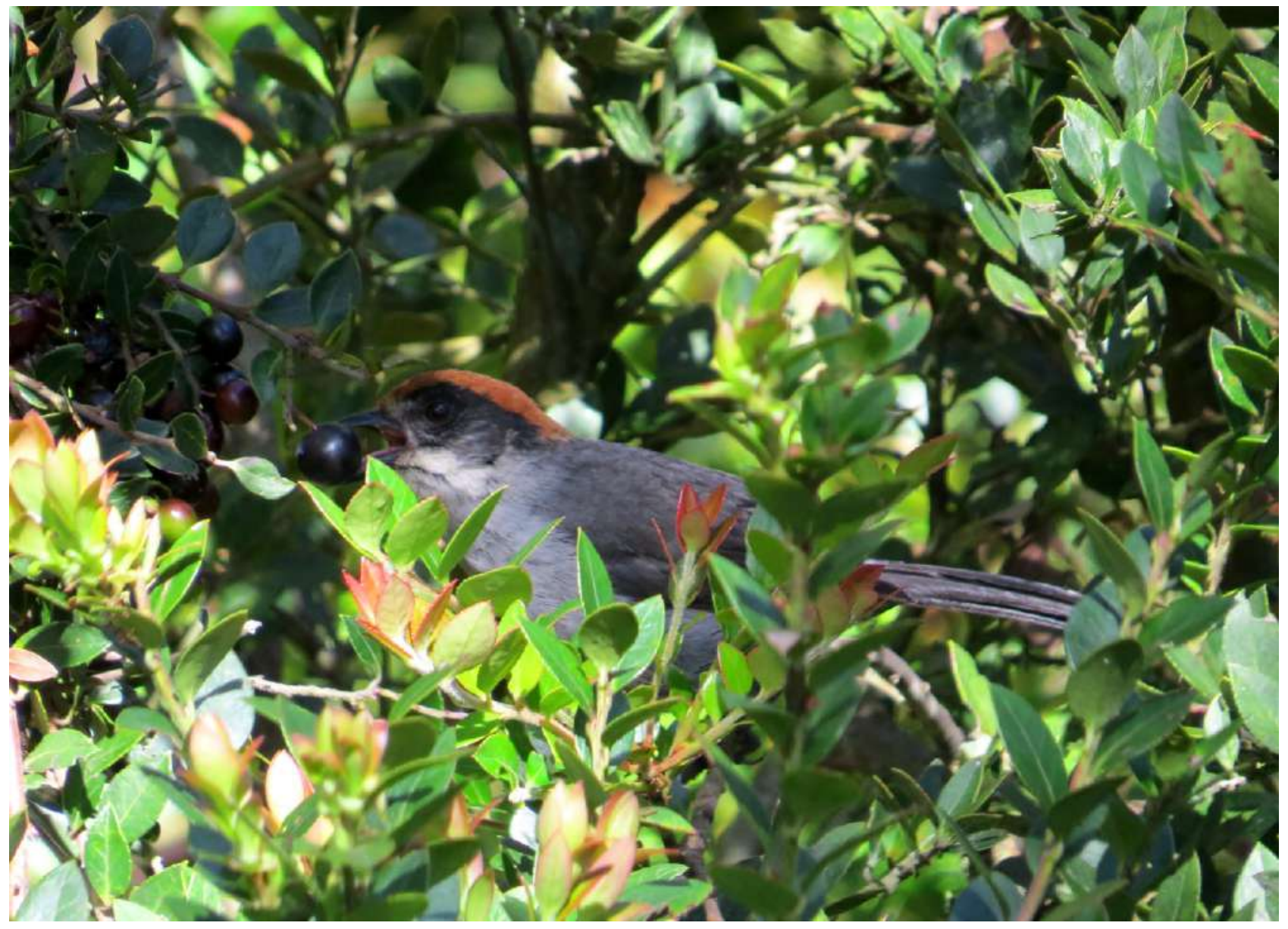

Figura 4. Individuo de Atlapetes blancae consumiendo Vaccinium meridionale, en el municipio de San Pedro de los Milagros, Antioquia, Colombia. Fotografía: Sergio Chaparro-Herrera. 
Por su lado, el sector denominado Llanos de Cuivá, del municipio de Yarumal, corresponde a una meseta de topografía irregular (2700 m s. n. m.), formada por ondulaciones del terreno y cuya vegetación arbórea ha desaparecido y queda solamente una cubierta raquítica de arbustos, hierbas y helechos sobre un suelo erosionado y empobrecido y en cuyos pequeños valles aparece un paramillo (páramo azonal) sobre un terreno cenagoso, con abundancia de frailejones y otras plantas frecuentes en los páramos (Espinal, 2011) (figura 2C, 2D). Entre las plantas que podemos encontrar en este páramo azonal están (tomado de Espinal, 2011): Alchemilla orbiculata (Rosaceae), Baccharis tricuneata, Espeletia occidentalis, Monticalia ledifolia (Asteraceae), Blechnum sp. (Blechnaceae), Bomarea sp. (Alstroemeriaceae), Castilleja fissifolia (Orobanchaceae), Clethra sp. (Clethraceae), Chaetolepis microphylla (Melastomataceae), Chusquea sp. (Poaceae), Gaultheria sp. (Ericaceae), Geranium sp. (Geraniaceae), Hedyosmum sp. (Chlorantaceae), Hesperomeles ferruginea, Hypericum sp. (Hypericaceae), Isoetes sp. (Isoetaceae), Lycopodium clavatum (Lycopodiaceae), Monnina sp. (Polygalaceae), Myrica pubescens (Myricaceae), Paepalanthus dendroides (Eriocaulaceae), Campyloneurum angustifolium (Polypodiaceae), Rhamnus sp. (Rhamnaceae), Symplocos theiformis (Symplocaceae), Harperocallis sessiliflora (Todfieldiaceae), Weinmannia sp. (Cunoniaceae), Viburnum sp. (Viburnaceae), Xyris columbiana (Xyridaceae), junto con algunos relictos de Q. humboldtii (Fagaceae) que cubrían en el pasado una mayor extensión de estas tierras (Espinal, 2011). Adicionalmente, pudimos identificar en campo: Puya roldanii (Bromeliaceae), H. obtusifolia (Rosaceae), Psychotria sp. (Rubiaceae), B. nitida, D. alaternoides, G. erecta (Ericaceae) y orquídeas (Orchidaceae) como Maxillaria aurea, Elleantus maculatus y Cyrtochilum amphiglottis (ver Dalström, 2020 para taxonomía de esta última orquídea).

\section{Notas para la conservación del hábitat de A. blan- cae}

Las estrategias de conservación de $A$. blancae incluyen conservar parches de vegetación nativa de matorral donde todavía se encuentra presente. Asimismo, ampliar el área que estos ocupan, buscando conectar relictos cercanos y aumentar el área total de hábitat adecuado y conectado disponible para las aves. Es importante mencionar que, en el municipio de San Pedro de los Milagros, algunas áreas donde se ha registrado $A$. blancae, corresponden a sitios donde hace 50-100 años se practicó la minería de oro, actividad que, luego de la tala de los antiguos robledales, pudo haber causado la acidificación y empobrecimiento del suelo. Las nuevas características del suelo han permitido que, una vez abandonada la actividad minera, se establezca en estos lugares la vegetación resistente de matorrales mencionada anteriormente.

En términos de restauración ecológica, afortunadamente el matorral que parece preferir $A$. blancae como hábitat, es relativamente bastante rápido y fácil de restaurar, en comparación con lo que tardaría un bosque. En la mayor parte de las áreas es necesario identificar los lugares clave para realizar procesos de conectividad y llevar a cabo acuerdos con los propietarios para asegurar que estos corredores puedan ser establecidos y mantenidos en el corto, mediano y largo plazo, procesos sociales clave en la restauración ecológica (Aguilar-Garavito y Ramírez, 2016; Ospina et al., 2015; Vargas, 2007). Uno de los pasos más importantes en esta primera etapa es la instalación de cercas protectoras, para evitar que el ganado ingrese y permitir que el pasto en estas áreas crezca, sin volver a cortarlo de nuevo, y con ello propender el desarrollo de procesos de regeneración natural de la vegetación. Vale la pena resaltar que, si existen relictos de vegetación nativa alrededor de estas áreas a restaurar, el área pronto presentará helechos y en él crecerá una serie de arbustos pioneros de crecimiento rápido. Es probable que, en muchos casos, esta regeneración ayude a crear nuevos relictos y corredores de hábitat adecuados para A. blancae, como ha sucedido en proyectos de conservación de especies afines, como el Matorralero Cabecipálido (Atlapetes pallidiceps) (Krabbe, 2004).

Por otro lado, en los casos donde la restauración se pretenda realizar en áreas con alto nivel de transformación y en donde no existen relictos cercanos de vegetación nativa, o cuando se desee acelerar la regeneración del hábitat de $A$. blancae, se debe enriquecer con más especies su hábitat, plantando especies nativas, preferiblemente propagadas en vive- 
ros locales y especialmente especies leñosas, haciendo énfasis en aquellas pioneras cuyo crecimiento es más veloz en terrenos abiertos y especies que se han registrado como alimentos importantes para la $A$. blancae (anexo 1).

Un caso similar sucede en Ecuador, en donde se empelaron y sugirieron diferentes estrategias para la protección de Atlapetes pallidiceps y su hábitat. Dentro de estas se encuentra el manejo del hábitat, en áreas donde la vegetación es demasiado densa y alta, esto mediante la tala selectiva con el objetivo de eliminar la vegetación de más de tres metros y preservar un máximo de diez árboles pequeños y dos árboles grandes en cada territorio (Krabbe, 2004), así como mantener de manera controlada y rotativa la presión de pastoreo, en niveles bajos a intermedios, para preservar los matorrales densos de los que depende esta especie, sumado a la eliminación de la vegetación leñosa para evitar la invasión de árboles y a la siembra de vegetación de matorral en áreas donde ya se han realizado actividades agrícolas y que se encuentran abandonadas (Hartmann et al., 2015; Oppel et al., 2004). Finalmente, se menciona para esta especie y al igual que señalamos para $A$. blancae, que el plan de conservación debe enfocarse en la protección de fragmentos de matorral con características apropiadas, junto con procesos de conectividad para facilitar el intercambio genético (Oppel et al., 2004).

Las observaciones mencionadas anteriormente junto con los comentarios de hábitat y restauración pretenden, además de aportar al conocimiento de la biología de la especie, brindar información que permita involucrar la vegetación empleada por $A$. blancae con procesos de restauración ecológica en el área de distribución. Lo anterior con el objetivo de tomar acciones de conservación que deberían ser incorporadas e implementadas en el Sistema Local de Áreas Protegidas (SILAP) existentes en la región (en el caso de San Pedro de los Milagros y Santa Rosa de Osos), y cualquier otra figura legal. Además de incentivar iniciativas privadas como el desarrollo de sistemas silvopastoriles y ganadería sostenible, entre otros, que conlleven a tomar acciones de protección y evitar la pérdida de hábitat de la especie a futuro. Esto solo puede ser efectivo al existir una articulación de entidades de acción en el territorio, ya sea de carácter público o privado, junto con actores locales tomadores de decisiones.

\section{AGRADECIMIENTOS}

A The Association of Field Ornithologists (AFO) y Neotropical Bird Club (NBC) por la financiación y apoyo al Proyecto Atlapetes, y a Esteban Domínguez por la identificación de algunas orquídeas.

\section{CONFLICTO DE INTERESES}

Los autores declaran no tener conflictos de intereses.

\section{REFERENCIAS}

Administración Municipal. (2000). Esquema de ordenamiento territorial del municipio de San Pedro, acuerdo 080 de 2000. San Pedro de los Milagros, Colombia: Administración Municipal San Pedro de los Milagros.

Aguilar-Garavito, M., \& Ramírez, W. (2016). Fundamentos y consideraciones generales sobre restauración ecológica para Colombia. Biodiversidad en la Práctica 1(1), 147-176. http://revistas.humboldt.org.co/index. $\mathrm{php} / \mathrm{BEP} /$ article/view/50.

Arias, L. A. (1995). El relieve de la zona central de Antioquia: un palimpsesto de eventos tectónicos y climáticos. Revista Facultad de Ingeniería, Universidad de Antioquia, 10, 9-24. https://revistas.udea.edu.co/index.php/ ingenieria/article/view/325539.

Arias, L. A., González, L. H., \& Arias, G. (2000). Historia del Relieve y los Suelos en el Altiplano de Santa Rosa de Osos Antioquia Región el Vergel. Universidad Nacional de Colombia-Sede Medellín y Corantioquia. https://cia.corantioquia.gov.co/cgi-bin/ koha/opac-detail.pl?biblionumber $=4977$

Arias, L. A., Parra, N., González, L. H., Cabrera, K., \& Zapata, R. (2006). Cartografía del relieve en el altiplano de Santa Rosa de Osos. Universidad Nacional de Colombia-sede Medellín. https://repositorio.unal.edu. co/handle/unal/55873.

Arias, L. A., \& Gonzáles, L. H. (2007). Morfología del altiplano de Santa Rosa de Osos (ASRO). Boletín de Geología, 29(1), 95-102. DOI:10.18273/revbol.

Arias, L. A. (2011). Estructura, Clasificación y Evolución del Relieve en el Departamento de Antioquia. En R. Callejas, A. Idárraga (Eds.). Flora de Antioquia: catálogo de las plantas vasculares. Introducción. (vol. I, pp. 19-180). Universidad de Antioquia, Missouri Botanical Garden y Oficina de Planeación Departamental de la Gobernación de Antioquia. https://www.academia.edu/13952345/flora de_antioquia_catalogo_de_las_plantas_vasculares

Avellaneda-Cadena, C., \& Betancur, J. (2007). Las plantas vasculares de los afloramientos rocosos de Suesca, Cordillera 
Oriental colombiana. Actualidades Biológicas, 29(86), 25-39. https://revistas.unal.edu.co/index.php/actabiol/ article/view/27547.

BirdLife International. (2018, noviembre 12). Atlapetes blancae. IUCN Red List of threatened species. DOI:10.2305/IUCN.UK.20163.RLTS.T22735460A95111951.en

Chaparro-Herrera, S., Echeverry-Galvis, M. Á., CórdobaCórdoba, S., \& Sua-Becerra, A. (2013). Listado actualizado de las aves endémicas y casi-endémicas de Colombia. Biota Colombiana, 14, 235-272. http://revistas. humboldt.org.co/index.php/biota/article/view/289.

Chaparro-Herrera, S., \& Lopera-Salazar, A. (2019). Descripción del nido, huevos y comportamientos reproductivos del Gorrión-montés paisa (Atlapetes blancae). Ornitología Colombiana, 17(eNB08), 1-6. https://asociacioncolombianadeornitologia.org/descripc ion-del-nido-huevos-comportamientos-reproductivos-delgorrion-montes-paisa-atlapetes-blancae/

Correa, R., Chaparro-Herrera, S., Lopera-Salazar, A., \& Parra, J. L. (2019). Redescubrimiento del Gorrión-Montés Paisa Atlapetes blancae. Cotinga, 41, 101-108. https://www. researchgate.net/publication/332913121.

Dalström, S. (2020). New combinations in Cyrtochilum (Orchidaceae: Oncidiinae). Lankesteriana, 20(1), 21-29. DOI:10.15517/LANK.V20I1.41110.

Donegan, T. M. (2007). A new species of brush finch (Emberizidae: Atlapetes) from the northern Central Andes of Colombia. Bulletin of the British Ornithologists' Club, 127, 255-268. https://www.biodiversitylibrary.org/page/ 40881795\#page/5/mode/1up.

Donegan, T. M., Avendaño, J. E., Huertas, B., \& Flórez, P. (2009). Avifauna de San Pedro de los Milagros, Antioquia: una comparación entre colecciones antiguas y evaluaciones rápidas. Boletín Científico. Centro de Museos. Museo de Historia Natural, 13, 63-72. http://vip.ucaldas. edu.co/boletincientifico/downloads/Boletin13(1)_5.pdf.

Espinal, L.S. (2011). Zonas de vida del departamento de Antioquia. En: R. Callejas, \& A. Idárraga (Eds.). Flora de Antioquia: catálogo de las plantas vasculares. Introducción. (vol. I, pp. 235-289). Universidad de Antioquia, Missouri Botanical Garden, y Oficina de Planeación Departamental de la Gobernación de Antioquia. https://www.academia.edu/13952345/flora_de_ antioquia_catalogo_de_las_plantas_vasculares

Fernández-Méndez, F., Velasco-Salcedo, V. M., GuerreroContecha, J., Galvis, M., \& Neri, A. V. (2016). Recuperación ecológica de áreas afectadas por un incendio forestal en la microcuenca Tintales (Boyacá, Colombia). Colombia Forestal, 19(2), 143-160. DOI:10.14483/udistrital.jour.colomb.for.2016.2.a02.

Ferreyra, M., Cingolani, A., Ezcurra, C., \& Bran, D. (1998). High-Andean vegetation and environmental gradients in northwestern Patagonia, Argentina. Journal of Vegetation Sciencie, 9, 307-316. DOI:10.2307/3237095.

Hartmann, S. A., Segelbacher, G., Juiña, M. E., \& Schaefer, H. M. (2015). Effects of habitat management can vary over time during the recovery of an endangered bird species. Biological Conservation, 192, 154-160. DOI:10.1016/j.biocon.2015.09.014.
Holdridge, L. R. (1967). Life zone ecology. Tropical Science Center. http://reddcr.go.cr/sites/default/ files/centro-de-documentacion/holdridge_1966_-_life_ zone_ecology.pdf

Idárraga, A., Ortiz, R. del C., Callejas, R., \& Merello, M. (Eds.). (2011). Flora de Antioquia: catálogo de las plantas vasculares (vol. II). Listado de las plantas vasculares del departamento de Antioquia. Programa Expedición Antioquia-2103. Series Biodiversidad y Recursos Naturales. Universidad de Antioquia, Missouri Botanical Garden \& Oficina de planeación departamental de la gobernación de Antioquia, Editorial DVinni. http://www.missouribotanicalgarden.org/Portals/0/ staff/PDFs/ortiz/OrtizR2011Menispermaceae_Ant.pdf

Krabbe, N. (2004). Pale-headed Brush-finch Atlapetes pallidiceps: notes on population size, habitat, vocalizations, feeding, interference competition and conservation. Bird Conservation International, 14(2), 77-86. DOI:10.1017/S0959270904000115

Lees, A. C., Rosenberg, K. V., Ruiz-Gutiérrez, V., Marsden, S., Schulenberg, T. S., \& Rodewald, A. D. (2020). A roadmap to identifying and filling shortfalls in Neotropical ornithology. The Auk, 137(4), 1-17. DOI:10.1093/auk/ukaa048.

Oppel, S., Schaefer, H. M., Schmidt, V., \& Schröder, B. (2004). Habitat selection by the pale-headed brush-finch (Atlapetes pallidiceps) in southern Ecuador: implications for conservation. Biological Conservation, 118(1), 33-40. DOI:10.1016/j.biocon.2003.07.006

Ospina, O.L., Vanegas, S., Escobar, G.A., Ramírez, W., \& Sánchez, J.J. (2015). Plan Nacional de Restauración: restauración ecológica, rehabilitación y recuperación de áreas disturbadas. Ministerio de Ambiente y Desarrollo Sostenible. https://www.minambiente.gov.co/index. php/bosques-biodiversidad-y-servicios-ecosistematicos/ gestion-en-biodiversidad/restauracion-ecologica.

Renjifo, L. M., Gómez, M. F., Velásquez-Tibatá, J., AmayaVillarreal, A. M., Kattan, G. H., Amaya-Espinel, J. D. \& Burbano-Girón, J. (Eds.). (2014). Libro rojo de aves de Colombia 1. Bosques Húmedos de los Andes y la Costa Pacífica. Pontificia Universidad Javeriana e Instituto Alexander von Humboldt. http://www. humboldt.org.co/es/estado-de-los-recursos-naturales/ item/707-libro-rojoaves1.

Salamanca, B., \& Camargo, G. (2000). Protocolo Distrital de Restauración Ecológica. Departamento Técnico Administrativo Medio Ambiente (DAMA), Alcaldía Mayor de Bogotá D. C. http://www.ambientebogota.gov.co/documents/10157/ 200413/PROTOCOLO+DISTRITAL+SDA.pdf.

Toro, J. L. (2010). Árboles de las montañas de Antioquia. Corantioquia. https://www.corantioquia.gov.co/ SiteAssets/Lists/Administrar\%20Contenidos/ EditForm/ArbolesWeb.pdf.

Vargas, O. (Ed.). (2007). Guía metodológica para la restauración ecológica del bosque altoandino. Universidad Nacional de Colombia. https://www.researchgate.net/ publication/259482462_Guia_Metodologica_para_la_ Restauracion_Ecologica_del_bosque_altoandino.

Villareal, H. M., Álvarez, M., Córdoba-Córdoba, S., Esco- 
bar, F., Fagua, G., Gast, F., Mendoza, H., Ospina, M., \& Umaña, A.M. (2006). Manual de métodos para el desarrollo de inventarios de Biodiversidad. Instituto de Investigación de Recursos Biológicos Alexander von Humboldt. http://repository.humboldt.org.co/ bitstream/handle/20.500.11761/31419/63.pdf 
Anexo 1. Especies de plantas consumidas por Atlapetes blancae en tres municipios del Altiplano de Santa Rosa de Osos (San Pedro de los Milagros, Santa Rosa de Osos y Yarumal sector Llanos de Cuivá). La información consignada en la tabla corresponde a datos tomados de Idárraga et al. 2011. Zona de vida: bosque húmedo Montano Bajo (bh-MB), bosque húmedo Pre-Montano (bh-PM), bosque húmedo Tropical (bh-T), bosque muy húmedo Montano Bajo (bmh-MB), bosque muy húmedo Pre-Montano (bmh-PM), bosque muy húmedo Tropical (bmh-T) bosque pluvial Montano Bajo (bp-MB), bosque pluvial Pre-Montano (bp-PM) y bosque pluvial Montano (bp-M). En negrita nuevos registros (esta nota).

\begin{tabular}{|c|c|c|c|c|c|c|c|}
\hline Nombre científico & Localidad & Hábito & Origen & Zona de vida & Elevación & $\begin{array}{l}\text { Región fisiográfica en Antio- } \\
\text { quia }\end{array}$ & Municipios de registro \\
\hline Berberis medellinensis & $\begin{array}{l}\text { San Pedro de } \\
\text { los Milagros }\end{array}$ & Árbol & Nativa & $\begin{array}{l}\text { bmh-MB, } \\
\text { bp-M }\end{array}$ & $\begin{array}{l}3000-3500 \\
(2530)\end{array}$ & $\begin{array}{l}\text { Altiplanos de Santa Rosa de } \\
\text { Osos, Rionegro y Sonsón }\end{array}$ & $\begin{array}{l}\text { Bello, Belmira, Medellín, San } \\
\text { Pedro de los Milagros }\end{array}$ \\
\hline Weinmannia pubescens & $\begin{array}{l}\text { San Pedro de } \\
\text { los Milagros }\end{array}$ & $\begin{array}{l}\text { Arbusto, } \\
\text { subar- } \\
\text { busto }\end{array}$ & Nativa & $\begin{array}{l}\text { bh-MB, bh- } \\
P M, \text { bmh-MB }\end{array}$ & $1500-3000$ & $\begin{array}{l}\text { Altiplanos de Santa Rosa de } \\
\text { Osos, Rionegro y Sonsón, } \\
\text { Cordillera Occidental, valle } \\
\text { del río Porce, vertiente } \\
\text { occidental de la Cordillera } \\
\text { Occidental, vertiente oriental } \\
\text { de la Cordillera Occidental }\end{array}$ & $\begin{array}{l}\text { Abejorral, Betania, Buriticá, } \\
\text { Caicedo, Caldas, Carmen de } \\
\text { Viboral, El Retiro, Envigado, } \\
\text { Frontino, Guarne, Ituango, } \\
\text { Jardín, La Ceja del Tambo, } \\
\text { Marinilla, Medellín, Nariño, } \\
\text { Peque, Rionegro, San Pedro } \\
\text { de los Milagros, Santa Rosa } \\
\text { de Osos, Sonsón, Urrao, } \\
\text { Yarumal }\end{array}$ \\
\hline Vaccinium meridionale & $\begin{array}{l}\text { San Pedro de } \\
\text { los Milagros }\end{array}$ & Arbusto & Nativa & $\begin{array}{l}\text { bh-MB, bmh- } \\
M B, \text { bmh-PM }\end{array}$ & $1500-2500$ & $\begin{array}{l}\text { Altiplanos de Santa Rosa de } \\
\text { Osos, Rionegro y Sonsón, } \\
\text { vertiente occidental de la } \\
\text { Cordillera Central }\end{array}$ & $\begin{array}{l}\text { Belmira, El Retiro, Guarne, } \\
\text { Medellín, Santa Rosa de Osos, } \\
\text { San Pedro de los Milagros }\end{array}$ \\
\hline Bejaria aestuans & $\begin{array}{l}\text { San Pedro de } \\
\text { los Milagros, } \\
\text { Santa Rosa de } \\
\text { Osos }\end{array}$ & Arbusto & Nativa & $\begin{array}{l}\text { bh-PM, bmh- } \\
\text { MB, bmh-PM, } \\
\text { bp-MB, bp-PM }\end{array}$ & $1500-3000$ & $\begin{array}{l}\text { Altiplanos de Santa Rosa de } \\
\text { Osos, Rionegro y Sonsón, } \\
\text { Cordillera Occidental, } \\
\text { vertiente occidental de la } \\
\text { Cordillera Central, vertiente } \\
\text { occidental de la Cordillera } \\
\text { Occidental, vertiente oriental } \\
\text { de la Cordillera Central }\end{array}$ & $\begin{array}{l}\text { Amalfi, Belmira, Caldas, } \\
\text { Carmen de Viboral, El Retiro, } \\
\text { Guarne, La Unión, Medellín, } \\
\text { Rionegro, San Pedro de los } \\
\text { Milagros, San Vicente, Santa } \\
\text { Bárbara, Santa Rosa de Osos, } \\
\text { Sonsón, Urrao }\end{array}$ \\
\hline Baccharis nitida & $\begin{array}{l}\text { San Pedro de } \\
\text { los Milagros, } \\
\text { Santa Rosa de } \\
\text { Osos, Llanos de } \\
\text { Cuivá (Yarumal) }\end{array}$ & Arbusto & Nativa & $\begin{array}{l}\text { bh-MB, bh- } \\
\text { PM, bmh-MB, } \\
\text { bp-PM }\end{array}$ & $\begin{array}{l}1000-2500 \\
(2837)\end{array}$ & $\begin{array}{l}\text { Altiplanos de Santa Rosa de } \\
\text { Osos, Rionegro y Sonsón, } \\
\text { Cordillera Occidental, } \\
\text { vertiente occidental de la } \\
\text { Cordillera Occidental }\end{array}$ & $\begin{array}{l}\text { Ciudad Bolívar, Dabeiba, } \\
\text { Frontino, Guarne, Jericó, La } \\
\text { Ceja del Tambo, La Unión, } \\
\text { Medellín, Nariño, Salgar, San } \\
\text { Pedro de los Milagros, Santa } \\
\text { Bárbara, Sonsón, Urrao, } \\
\text { Santa Rosa de Osos, Llanos } \\
\text { de Cuivá (Yarumal) }\end{array}$ \\
\hline
\end{tabular}




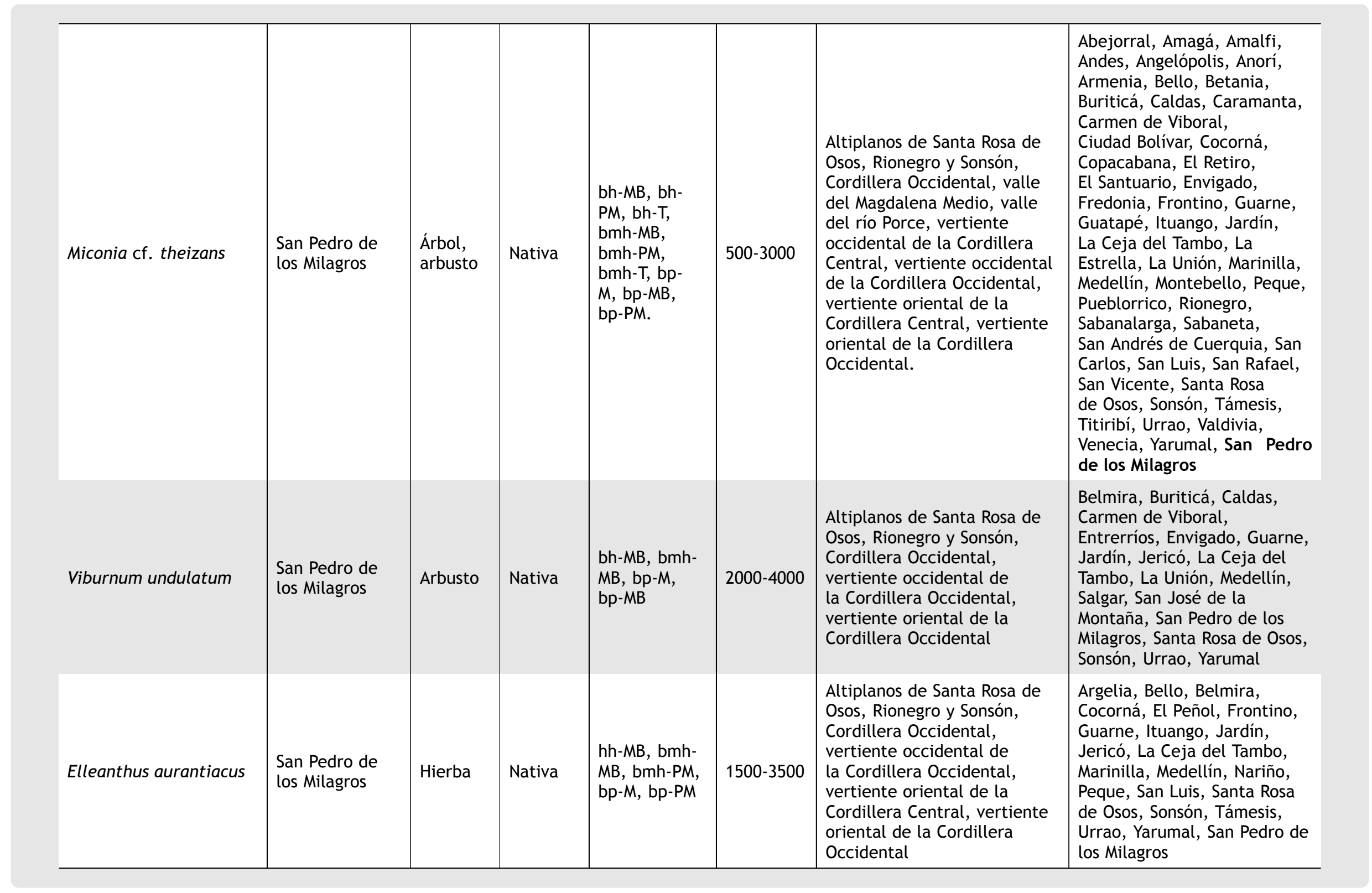




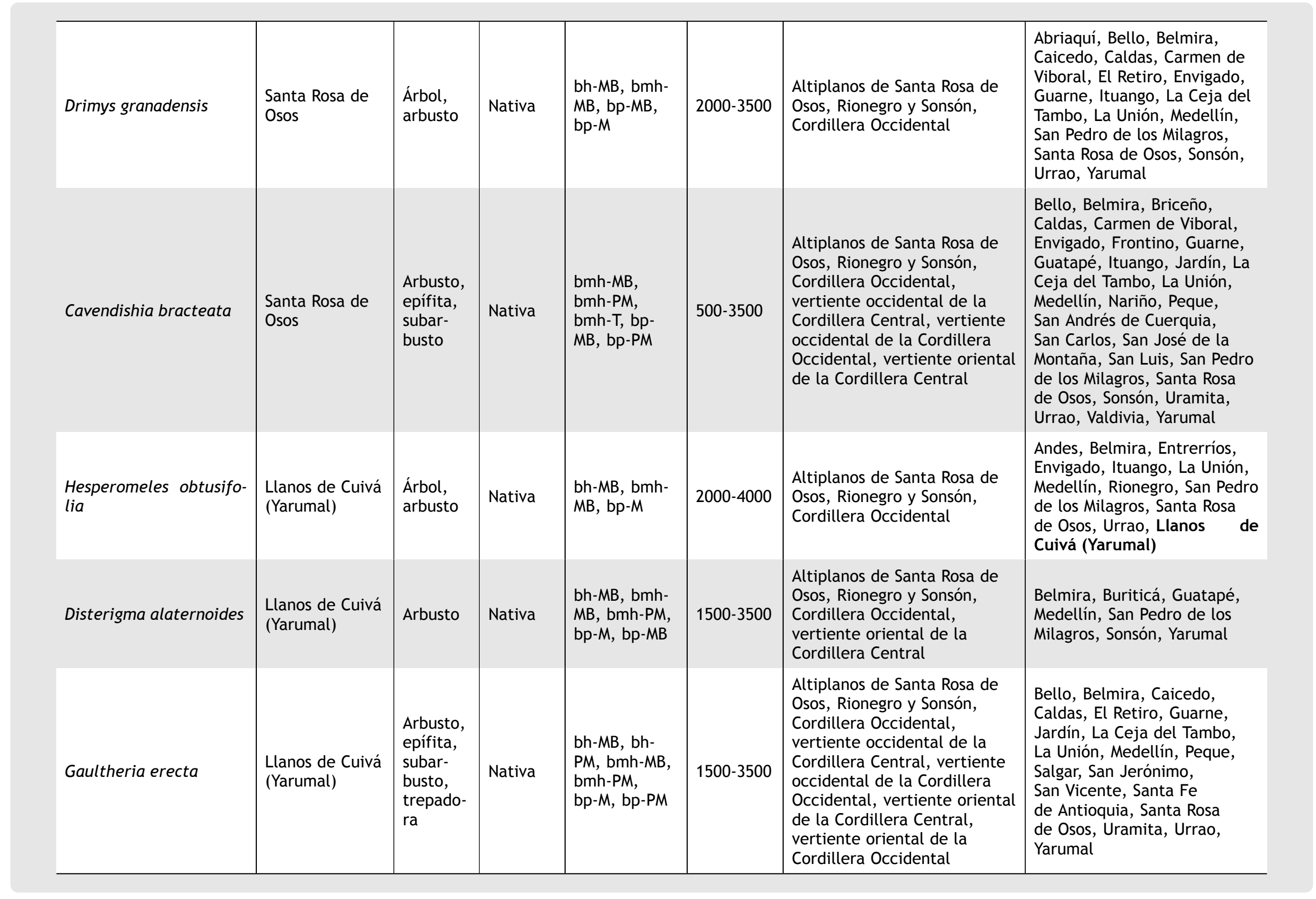




\begin{tabular}{|c|c|c|}
\hline Género & Localidad & Comentario \\
\hline Myrcianthes sp. & $\begin{array}{l}\text { San Pedro de } \\
\text { los Milagros }\end{array}$ & 4 especies reportadas para Antioquia \\
\hline Monnina sp. & $\begin{array}{l}\text { Santa Rosa de } \\
\text { Osos }\end{array}$ & 10 especies reportadas para Antioquia \\
\hline Psychotria sp. & $\begin{array}{l}\text { Llanos de Cuivá } \\
\text { (Yarumal) }\end{array}$ & 71 especies reportadas para Antioquia \\
\hline
\end{tabular}

